\title{
Conserving and gapless approximations for the composite bosons in terms of the constituent fermions
}

\author{
G.C. Strinati and P. Pieri \\ Dipartimento di Fisica, UdR INFM, Università di Camerino, I-62032 Camerino, Italy
}

(Dated: October 12, 2018)

\begin{abstract}
A long-standing problem with the many-body approximations for interacting condensed bosons has been the dichotomy between the "conserving" and "gapless" approximations, which either obey the conservations laws or satisfy the Hugenholtz-Pines condition for a gapless excitation spectrum, in the order. It is here shown that such a dichotomy does not exist for a system of composite bosons, which form as bound-fermion pairs in the strong-coupling limit of the fermionic attraction. By starting from the constituent fermions, for which conserving approximations can be constructed for any value of the mutual attraction according to the Baym-Kadanoff prescriptions, it is shown that these approximations also result in a gapless excitation spectrum for the boson-like propagators in the broken-symmetry phase. This holds provided the corresponding equations for the fermionic single- and two-particle Green's functions are solved self-consistently.

PACS numbers: PACS numbers: 03.75.Ss, 03.75.Hh, 05.30.Jp
\end{abstract}

Many-body decriptions of a system of interacting consensed bosons have long been known [1] to fall into either one of two classes of approximation schemes, which are alternatively consistent with the conservation laws (conserving approximations) or with the absence of a gap in the elementary excitations spectrum (gapless approximations). Having to choose between these two types of approximations constitutes a shortcoming of the manybody theory for condensed bosons, as one would rather like to deal with approximations which are conserving and gapless at the same time.

These approximation schemes have been conceived for interacting-boson systems like helium, for which the internal fermionic structure is immaterial, due to the large amount of energy required to excite the internal fermionic degrees of freedom compared with the energy scales of the experiments.

Recent experimental advances with ultracold trapped Fermi atoms have made it possible to produce systems of composite bosons (dimers), whose binding energy is comparable with the energy and temperature involved in the experiments 2]. The Bose-Einstein condensation of the dimers has also been detected [3]. In these systems, the internal fermionic structure is definitely relevant, as the binding energy of the dimers can be tuned across threshold via a Fano-Feshbach resonance [4], sweeping from bound to unbound fermions and viceversa.

For these systems, it appears appropriate to construct the dynamical propagators of the condensed composite bosons in terms of the constituent fermions, by following the progressive quenching of the fermionic degrees of freedom as the fermionic attraction is increased. For the constituent fermions, it is known that conserving approximations can be constructed for any value of the mutual attraction even in the broken-symmetry (superfluid) phase, via the Baym-Kadanoff prescriptions [5, 6] which require the self-consistent solution of the equations for the single- and two-particle Green's functions. In this way, conservation laws can be regarded to be fulfilled not only in terms of the constituent fermions but also in terms of the composite bosons, when the fermionic attraction gets sufficiently strong that the fermionic degrees of freedom (internal to the composite bosons) are progressively quenched.

The question is whether such conserving approximations (for the constituent fermions and therefore for the composite bosons) could also result into a gapless excitation spectrum for the propagators of the composite bosons in the broken-symmetry phase, the gapless condition being required on general grounds by the occurrence of a Goldstone mode 7]. [The case of a homogeneous system will be specifically considered when discussing the absence of a gap in the bosonic excitation spectrum [8].]

Purpose of this paper is to show that a given fermionic conserving approximation also results in a gapless excitation spectrum for the boson-like propagators. The long-standing dichotomy between conserving and gapless approximations can thus be apparently overcome, when the bosons themselves are treated at a more fundamental level in terms of the constituent fermions.

We begin by generalizing to the composite bosons the theorem of Hugenholtz-Pines for ordinary bosons, by following the treatment of Ref. 1 in terms of formally exact propagators (see also Ref. 7). Fermionic conserving approximations to select approximate propagators for the composite bosons will be considered later. To this end, we define a bosonic-like field operator

$$
\Psi_{B}(\mathbf{r})=\int d \boldsymbol{\rho} \phi(\rho) \psi_{\downarrow}(\mathbf{r}-\boldsymbol{\rho} / 2) \psi_{\uparrow}(\mathbf{r}+\boldsymbol{\rho} / 2)
$$

for any value of the fermionic coupling, where $\psi_{\sigma}(\mathbf{r})$ is a fermionic field operator with spin $\sigma$. When the fermionic attraction is sufficiently strong, on physical grounds the (real and normalized) function $\phi(\rho)$ can be taken as the 
bound solution of the associated two-body problem. At weaker coupling, a precise choice of $\phi(\rho)$ is not required. For instance, it could be taken as the solution of a generalized Cooper problem, whereby the Fermi energy is replaced by the coupling- (and temperature-) dependent fermionic chemical potential $\mu$. To break the gauge symmetry, the bosonic order parameter $\alpha(\mathbf{r})=\left\langle\Psi_{B}(\mathbf{r})\right\rangle_{\eta}$ is defined as the thermal average of the operator (1) within the restricted $(\eta)$ ensemble of Ref. 1. With the Nambu representation for the fermionic field operator $\left(\Psi_{1}(\mathbf{r})=\psi_{\uparrow}(\mathbf{r}), \Psi_{2}(\mathbf{r})=\psi_{\downarrow}^{\dagger}(\mathbf{r})\right)$, this thermal average can then be expressed in terms of the anomalous fermionic single-particle Green's function $\mathcal{G}_{12}$

$$
\left\langle\Psi_{2}^{\dagger}\left(\mathbf{r}-\frac{\boldsymbol{\rho}}{2}\right) \Psi_{1}\left(\mathbf{r}+\frac{\boldsymbol{\rho}}{2}\right)\right\rangle_{\eta}=\mathcal{G}_{12}\left(\mathbf{r}+\frac{\boldsymbol{\rho}}{2}, \mathbf{r}-\frac{\boldsymbol{\rho}}{2} ; \tau=0^{-}\right)
$$

with imaginary time $\tau$.

In what follows, it is convenient to consider a generalized fermionic single-particle Green's function:

$$
\mathcal{G}\left(1,1^{\prime}\right)=-\frac{\left\langle T_{\tau}\left[S \Psi(1) \Psi^{\dagger}\left(1^{\prime}\right)\right]\right\rangle}{\left\langle T_{\tau}[S]\right\rangle}
$$

with the notation $1=\left(\mathbf{r}_{1}, \tau_{1}, \ell_{1}\right)$ in terms of the Nambu spinor component $\ell$. Here, $T_{\tau}$ is the imaginary-timeordering operator, $\langle\cdots\rangle$ is a thermal average taken with the system grand-canonical Hamiltonian $K=H-\mu N$, $\Psi(1)=\exp \left\{K \tau_{1}\right\} \Psi_{\ell_{1}}\left(\mathbf{r}_{1}\right) \exp \left\{-K \tau_{1}\right\}$, and the operator $S=\exp \left\{-\int d 11^{\prime} \Psi^{\dagger}(1) U\left(1,1^{\prime}\right) \Psi\left(1^{\prime}\right)\right\}$ contains the source term

$U\left(1,1^{\prime}\right)=\left(\begin{array}{cc}U_{n}\left(\mathbf{r}_{\mathbf{1}}, \mathbf{r}_{\mathbf{1}^{\prime}} ; \tau_{1}\right) & U_{s}\left(\mathbf{r}_{1^{\prime}}, \mathbf{r}_{\mathbf{1}} ; \tau_{1}\right)^{*} \\ U_{s}\left(\mathbf{r}_{\mathbf{1}}, \mathbf{r}_{\mathbf{1}^{\prime}} ; \tau_{1}\right) & -U_{n}\left(\mathbf{r}_{\mathbf{1}}, \mathbf{r}_{1^{\prime}} ; \tau_{1}\right)\end{array}\right) \delta\left(\tau_{1}-\tau_{1^{\prime}}^{+}\right)$

with a normal $\left(U_{n}\right)$ and a superfluid $\left(U_{s}\right)$ component. [The normal component $U_{n}$ will not be needed in the following, while the superfluid component $U_{s}$ will be allowed to vanish at the end of the calculation.] In the static case (when $U$ does not depend on the imaginary time), the generalized definition (3) concides with the ordinary definition [like Eq.(2)] within the $\eta$-ensemble. With the definition (3), the bosonic order parameter is generalized as follows:

$$
\alpha(\mathbf{r})=\int d \boldsymbol{\rho} \phi(\rho) \mathcal{G}_{12}\left(\mathbf{r}+\frac{\boldsymbol{\rho}}{2}, \tau ; \mathbf{r}-\frac{\boldsymbol{\rho}}{2}, \tau^{+}\right) .
$$

Suppose now that $U_{s}\left(\mathbf{r}, \mathbf{r}^{\prime} ; \tau\right)$ is varied by a small uniform change of phase $\delta \Phi$, such that $\delta U_{s}\left(\mathbf{r}, \mathbf{r}^{\prime} ; \tau\right) \cong$ $i \delta \Phi U_{s}\left(\mathbf{r}, \mathbf{r}^{\prime} ; \tau\right)$. This change can be reabsorbed by a canonical tranformation of the fermionic field operators, so that the corresponding change of the order parameter (5) is given by $\delta \alpha(\mathbf{r})=-i \delta \Phi \alpha(\mathbf{r})$ to the leading order in $\delta \Phi$. The change $\delta \alpha(\mathbf{r})$ can be calculated alternatively via the definitions (5) and (3), by performing the functional derivative of (3) with respect to a variation of $U_{s}$. One obtains:

$$
\delta \alpha(\mathbf{r})=-\int d \boldsymbol{\rho} \phi(\rho) \int d \mathbf{r}_{\mathbf{2}} d \mathbf{r}_{\mathbf{2}}^{\prime} \int d \tau_{2}
$$

$$
\begin{aligned}
\times[L(\mathbf{r} & \left.+\frac{\boldsymbol{\rho}}{2}, \tau, 1 ; \mathbf{r}_{\mathbf{2}}, \tau_{2}, 2 ; \mathbf{r}-\frac{\boldsymbol{\rho}}{2}, \tau^{+}, 2 ; \mathbf{r}_{\mathbf{2}}^{\prime}, \tau_{2}^{+}, 1\right) \\
& \times \delta U_{s}\left(\mathbf{r}_{\mathbf{2}}, \mathbf{r}_{\mathbf{2}}^{\prime} ; \tau_{2}\right)^{*} \\
+L(\mathbf{r}+ & \left.\frac{\boldsymbol{\rho}}{2}, \tau, 1 ; \mathbf{r}_{\mathbf{2}}, \tau_{2}, 1 ; \mathbf{r}-\frac{\boldsymbol{\rho}}{2}, \tau^{+}, 2 ; \mathbf{r}_{\mathbf{2}}^{\prime}, \tau_{2}^{+}, 2\right) \\
& \left.\times \delta U_{s}\left(\mathbf{r}_{\mathbf{2}}^{\prime}, \mathbf{r}_{\mathbf{2}} ; \tau_{2}\right)\right] .
\end{aligned}
$$

Here, $L\left(1,2,1^{\prime}, 2^{\prime}\right)=\mathcal{G}_{2}\left(1,2,1^{\prime}, 2^{\prime}\right)-\mathcal{G}\left(1,1^{\prime}\right) \mathcal{G}\left(2,2^{\prime}\right)$ is the two-particle correlation function expressed in terms of the generalized fermionic two-particle Green's function

$$
\mathcal{G}_{2}\left(1,2,1^{\prime}, 2^{\prime}\right)=\frac{\left\langle T_{\tau}\left[S \Psi(1) \Psi(2) \Psi^{\dagger}\left(2^{\prime}\right) \Psi^{\dagger}\left(1^{\prime}\right)\right]\right\rangle}{\left\langle T_{\tau}[S]\right\rangle}
$$

and is obtained via the functional derivative $L\left(1,2,1^{\prime}, 2^{\prime}\right)=-\delta \mathcal{G}\left(1,1^{\prime}\right) / \delta U\left(2^{\prime}, 2\right)$.

By a similar token, for the adjoint $\alpha(\mathbf{r})^{*}$ of $\alpha(\mathbf{r})$ one obtains $\delta \alpha(\mathbf{r})^{*}=i \delta \Phi \alpha(\mathbf{r})^{*}$, as well as

$$
\begin{aligned}
\delta \alpha(\mathbf{r})^{*} & =-\int d \boldsymbol{\rho} \phi(\rho) \int d \mathbf{r}_{\mathbf{2}} d \mathbf{r}_{\mathbf{2}}^{\prime} \int d \tau_{2} \\
\times[L(\mathbf{r} & \left.-\frac{\boldsymbol{\rho}}{2}, \tau, 2 ; \mathbf{r}_{\mathbf{2}}, \tau_{2}, 2 ; \mathbf{r}+\frac{\boldsymbol{\rho}}{2}, \tau^{+}, 1 ; \mathbf{r}_{\mathbf{2}}^{\prime}, \tau_{2}^{+}, 1\right) \\
& \times \delta U_{s}\left(\mathbf{r}_{\mathbf{2}}, \mathbf{r}_{\mathbf{2}}^{\prime} ; \tau_{2}\right)^{*} \\
+L(\mathbf{r} & \left.-\frac{\boldsymbol{\rho}}{2}, \tau, 2 ; \mathbf{r}_{\mathbf{2}}, \tau_{2}, 1 ; \mathbf{r}+\frac{\boldsymbol{\rho}}{2}, \tau^{+}, 1 ; \mathbf{r}_{\mathbf{2}}^{\prime}, \tau_{2}^{+}, 2\right) \\
& \left.\times \delta U_{s}\left(\mathbf{r}_{\mathbf{2}}^{\prime}, \mathbf{r}_{\mathbf{2}} ; \tau_{2}\right)\right]
\end{aligned}
$$

in the place of (6). [The quantity $\alpha(\mathbf{r})^{*}$ is defined like in Eq.(5) with $\mathcal{G}_{21}$ replacing $\mathcal{G}_{12}$, and coincides with the complex conjugate of $\alpha(\mathbf{r})$ in the static case.]

The static and uniform limit of the above results can be considered at this point. Accordingly, without loss of generality we let $U_{s}\left(\mathbf{r}, \mathbf{r}^{\prime} ; \tau\right) \rightarrow U_{s} \phi\left(\left|\mathbf{r}-\mathbf{r}^{\prime}\right|\right)$ in both Eqs. (6) and (8), where $U_{s}$ is a (complex) constant and $\phi(\mathbf{r})$ the same function of Eq.(11). We also introduce the Fourier representation:

$$
\begin{aligned}
& L\left(1,2,1^{\prime}, 2^{\prime}\right)=\int \frac{d \mathbf{p}}{(2 \pi)^{3}} \frac{1}{\beta} \sum_{n} \int \frac{d \mathbf{p}^{\prime}}{(2 \pi)^{3}} \frac{1}{\beta} \sum_{n^{\prime}} \\
& \times \int \frac{d \mathbf{q}}{(2 \pi)^{3}} \frac{1}{\beta} \sum_{\nu} e^{i(\mathbf{p}+\mathbf{q}) \cdot \mathbf{r}_{1}} e^{i \mathbf{p}^{\prime} \cdot \mathbf{r}_{2}} e^{-i \mathbf{p} \cdot \mathbf{r}_{1}^{\prime}} e^{-i\left(\mathbf{p}^{\prime}+\mathbf{q}\right) \cdot \mathbf{r}_{\mathbf{2}}^{\prime}} \\
& \times e^{-i\left(\omega_{n}+\Omega_{\nu}\right) \tau_{1}} e^{-i \omega_{n^{\prime}} \tau_{2}} e^{i \omega_{n} \tau_{1}^{\prime}} e^{i\left(\omega_{n^{\prime}}+\Omega_{\nu}\right) \tau_{2}^{\prime}} L_{\ell_{1}^{\ell_{2} \ell_{2}}}^{\ell_{2}^{\prime}}\left(p, p^{\prime} ; q\right) .
\end{aligned}
$$

Here, $\mathbf{p}, \mathbf{p}^{\prime}$, and $\mathbf{q}$ are wave vectors, $\omega_{n}=(2 n+1) \pi / \beta$ ( $n$ integer) is a fermionic Matsubara frequency ( $\beta$ being the inverse temperature), $\Omega_{\nu}=2 \pi \nu / \beta$ ( $\nu$ integer) a bosonic Matsubara frequency, and $p=\left(\mathbf{p}, \omega_{n}\right)$, $p^{\prime}=\left(\mathbf{p}^{\prime}, \omega_{n^{\prime}}\right)$, and $q=\left(\mathbf{q}, \Omega_{\nu}\right)$ is a four-vector notation. By straightforward manipulations of Eqs. (6) and (8), and by recalling the identities $\delta \alpha(\mathbf{r})=-i \delta \Phi \alpha(\mathbf{r})$ and $\delta U_{s}\left(\mathbf{r}, \mathbf{r}^{\prime} ; \tau\right)=i \delta \Phi U_{s}\left(\mathbf{r}, \mathbf{r}^{\prime} ; \tau\right)$ (plus their adjoints), one ends up with the matrix equation

$$
\left(\begin{array}{c}
\alpha \\
-\alpha
\end{array}\right)=\left(\begin{array}{cc}
G_{22}^{11}(q \rightarrow 0) & G_{21}^{12}(q \rightarrow 0) \\
G_{12}^{21}(q \rightarrow 0) & G_{11}^{22}(q \rightarrow 0)
\end{array}\right)\left(\begin{array}{c}
U_{s} \\
-U_{s}
\end{array}\right)
$$


for $U$ and $\alpha$ real, with the notation

$$
\begin{aligned}
G_{\ell_{1}^{\prime} \ell_{2}}^{\ell_{1} \ell_{2}^{\prime}}(q) & =-\int \frac{d \mathbf{p}}{(2 \pi)^{3}} \frac{1}{\beta} \sum_{n} e^{i \omega_{n} 0^{+}} \int \frac{d \mathbf{p}^{\prime}}{(2 \pi)^{3}} \frac{1}{\beta} \sum_{n^{\prime}} e^{i \omega_{n^{\prime}} 0^{+}} \\
& \times \phi(\mathbf{p}+\mathbf{q} / 2) \phi\left(\mathbf{p}^{\prime}+\mathbf{q} / 2\right) L_{\ell_{1}^{\prime} \ell_{2}}^{\ell_{1} \ell_{2}^{\prime}}\left(p, p^{\prime} ; q\right)
\end{aligned}
$$

It can be readily verified that, in the limit $U_{s} \rightarrow 0$, the definition (11) corresponds to the four possible (normal and anomalous) bosonic-like propagators which can be constructed with the operator (1) and its adjoint.

Before letting $U_{s} \rightarrow 0$ in Eq. (10), it is convenient to introduce the inverse of the matrix on its right-hand side and write

$$
\left(\begin{array}{ll}
G_{22}^{11}(q \rightarrow 0) & G_{21}^{12}(q \rightarrow 0) \\
G_{12}^{21}(q \rightarrow 0) & G_{11}^{22}(q \rightarrow 0)
\end{array}\right)=\frac{1}{A D-B C}\left(\begin{array}{cc}
D & -B \\
-C & A
\end{array}\right) .
$$

Matrix inversion of Eq.(10) then yields the conditions

$$
A-B=0 \quad, \quad C-D=0,
$$

in order to have a finite value for the order parameter $\alpha$ in the limit $U_{s} \rightarrow 0$. These conditions are not independent from each other, since one can prove on general ground from time-reversal invariance that $A=D$ and $B=C$. The denominator in Eq. (12) then reduces to $A D-B C=(A-B)(A+B)$, and vanishes owing to (13). This implies that the bosonic-like propagators (11) are singular when $q \rightarrow 0$, irrespective of the value of the fermionic coupling. In the present context, the condition $A-B=0$ corresponds to the Hugenholtz-Pines theorem for ordinary bosons [9]. It implies, in particular, that the composite bosons, which form when the fermionic attraction is strong enough, have a gapless spectrum.

All considerations made so far hold for the exact fermionic single- [Eq.(3)] and two-particle [Eq.(7)] Green's functions. The crucial point to derive the result (10) was that the single- and two-particle fermionic Green's functions are related to each other via a functional differentiation in the presence of the external potential $U$. When dealing with fermions, it is standard practice to explore this relation further [5, 6], by exploiting the Dyson's equation with the self-energy $\Sigma$ :

$$
-\mathcal{G}^{-1}(1,2)=\frac{\partial}{\partial \tau_{1}} \delta(1,2)+M(1,2)+U(1,2)+\Sigma(1,2) .
$$

In this expression, $M(1,2)=\tau_{\ell_{1} \ell_{2}}^{3}\left(h\left(\mathbf{r}_{1}\right)-\mu\right) \delta\left(\mathbf{r}_{1}-\right.$ $\left.\mathbf{r}_{\mathbf{2}}\right) \delta\left(\tau_{1}-\tau_{2}\right)$ where $\tau^{3}$ is a Pauli matrix and $h(\mathbf{r})$ is the single-particle Hamiltonian (which, in general, includes an external static potential). The two-particle correlation function $L$ is correspondingly obtained as:

$$
\begin{aligned}
& -L\left(1,2,1^{\prime}, 2^{\prime}\right)=\frac{\delta \mathcal{G}\left(1,1^{\prime}\right)}{\delta U\left(2^{\prime}, 2\right)} \\
& =-\int d 34 \mathcal{G}(1,3) \frac{\delta \mathcal{G}^{-1}(3,4)}{\delta U\left(2^{\prime}, 2\right)} \mathcal{G}\left(4,1^{\prime}\right)=\mathcal{G}\left(1,2^{\prime}\right) \mathcal{G}\left(2,1^{\prime}\right) \\
& +\int d 3456 \mathcal{G}(1,3) \mathcal{G}\left(6,1^{\prime}\right) \frac{\delta \Sigma(3,6)}{\delta \mathcal{G}(4,5)}(-) L\left(4,2,5,2^{\prime}\right) .(15)
\end{aligned}
$$

It thus satisfies the Bethe-Salpeter equation, with kernel $\delta \Sigma / \delta \mathcal{G}$ related to the kernel $\Sigma$ of the Dyson's equation (14). The limit $U \rightarrow 0$ can be taken in Eqs. (14) and (15) whenever appropriate.

Selection of an approximate fermionic many-body theory starts with an approximate choice of the functional form of the self-energy $\Sigma$ in terms of $\mathcal{G}$ (and of the two-body interaction). The equations (14) and (15) are then solved self-consistently, with the respective approximate kernels $\Sigma$ and $\delta \Sigma / \delta \mathcal{G}$. In addition, Eq.(15) implies that Eq.(6) (and its adjoint (8) ) holds even for the approximate theory, since $L$ (with the approximate kernel $\delta \Sigma / \delta \mathcal{G}$ ) still represents the functional derivative of $\mathcal{G}$ with respect to $U$. The alternative result $\delta \alpha(\mathbf{r})=-i \delta \Phi \alpha(\mathbf{r})$ is instead obtained in the approximate theory by noting that, under the transformation $U_{s}\left(\mathbf{r}, \mathbf{r}^{\prime} ; \tau\right) \rightarrow e^{i \delta \Phi} U_{s}\left(\mathbf{r}, \mathbf{r}^{\prime} ; \tau\right)$, the approximate offdiagonal single-particle Green's function $\mathcal{G}_{12}$ of Eq. (5) trasforms as $\mathcal{G}_{12} \rightarrow e^{i \delta \Phi} \mathcal{G}_{12}$. As a consequence, the result (13) follows even within the approximate theory, implying a gapless spectrum.

Conserving approximations for fermions are similarly based on Eqs. (14) and (15), which are solved selfconsistently for a given approximate choice of $\Sigma$. In this case, the self-energy has to be chosen appropriately, to comply with the requirements of local number conservation and gauge invariance [10]. It is then required that the symmetry property $L\left(1,2,1^{\prime}, 2^{\prime}\right)=L\left(2,1,2^{\prime}, 1^{\prime}\right)$ is satisfied by the approximate $L$. To this end, it is sufficient that the approximate kernel $\delta \Sigma / \delta \mathcal{G}$ of Eq. (15) satisfies the same symmetry property. This property is, in turn, met by any $\Phi$-derivable approximation for the self-energy $\Sigma$ of Eq. (14), whereby $\Sigma(1,2)=\delta \Phi / \delta \mathcal{G}(2,1)$ is obtained from an approximate functional $\Phi$. [6] Attention must be paid to the fact that a given choice for $\Sigma$ may not meet this requirement, unless certain diagrams for $\Sigma$ are taken together [6, 11].

It is now evident that a conserving approximation for the constituent fermions, which holds for any value of their mutual attraction, will also result in a conserving approximation for the composite bosons that form in strong coupling. The same fermionic conserving approximation will further result in a gapless spectrum for the composite bosons, as the same requirement for Eqs. (14) and (15) to be simultaneously self-consistently satisfied applies to both (conserving and gapless) procedures. This proves our claim. From the above considerations, it is also clear that the requirements for a fermionic approximation to be conserving at any given coupling are more stringent than the absence of a gap in the excitation spectrum of the composite bosons in strong coupling.

A well-known example of a fermionic conserving approximation, which results for any coupling in a gapless spectrum for the collective mode associated with the broken symmetry 10], is the BCS (off-diagonal) approximation for $\Sigma$, shown in Fig.1(a) with the associated po- 


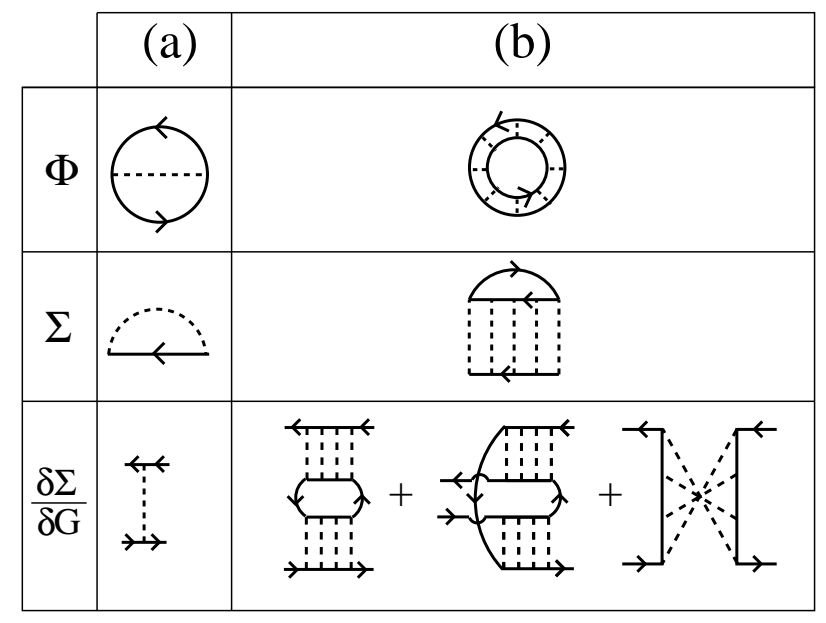

FIG. 1: Self-energy $\Sigma$ derived from the potential $\Phi$ with the associated kernel $\delta \Sigma / \delta \mathcal{G}$, for (a) the BCS approximation and (b) the t-matrix approximation in the broken-symmetry phase. Full lines represent fermionic (self-consistent) singleparticle Green's functions, with the arrows pointing from the second to the first argument; broken lines represent the fermionic interaction potential.

tential $\Phi$ and kernel $\delta \Sigma / \delta \mathcal{G}$ of the Bethe-Salpeter equation (15). In this case, the self-consistent solution of the Dyson's equation (14) reduces to the solution of the BCS gap equation; in addition, this equation coincides with the condition $A-B=0$ of Eq.(13) which guarantees the absence of a gap in the bosonic excitation spectrum. In this simple case, therefore, the self-consistent solution of the BCS gap equation is sufficient for the approximation to be conserving and gapless. More generally, separate solutions of the equations (14) and (15) are required for the approximation to be conserving and gapless. For instance, to the self-energy $\Sigma$ within the fermionic t-matrix approximation in the broken-symmetry phase [12], shown in Fig.1(b) with the associated potential $\Phi$, there correspond three distinct contributions to the kernel $\delta \Sigma / \delta \mathcal{G}$, also shown in Fig.1(b). When considering the BCS and t-matrix approximations for $\Sigma$ together, to get a gapless spectrum it is thus not enough to solve self-consistently the Dyson's equation for $\mathcal{G}$ with both self-energy contributions, if one solves at the same time the BetheSalpeter equation with only the BCS contribution to kernel $\delta \Sigma / \delta \mathcal{G}$. By doing so, one would, in fact, omit the three contributions to $\delta \Sigma / \delta \mathcal{G}$ depicted in Fig.1(b), whose presence is required by conserving criteria. Additional conserving and gapless approximations can be similarly constructed by suitable choices of the fermionic self-energy.

In conclusion, we have shown that a given conserving approximation for the constituent fermions also results into a gapless spectrum for the composite bosons. By following the formation of the bosons from the constituent fermions as the fermionic attraction is progressively increased, a long-standing (conserving vs gapless) dichotomy can thus be resolved, at least at a formal level. Although the self-consistent solution of the equations determining the fermionic single- and two-particle Green's functions might, in general, involve considerable numerical labor, enforcing the fermionic conserving criteria proves per se sufficient to get a gapless bosonic spectrum.

We are indebted to A. Fetter, F. Iachello, and G. Morandi for a critical reading of the manuscript. This work was supported by the Italian MIUR (contract Cofin2003 "Complex Systems and Many-Body Problems").

[1] P.C. Hohenberg and P.C. Martin, Ann. Phys. 34, 291 (1965).

[2] C.A. Regal, C. Ticknor, J.L. Bohn, and D.S. Jin, Nature 424, 47 (2003); K.E. Strecker, G.B. Partridge, and R.G. Hulet, Phys. Rev. Lett. 91, 080406 (2003).

[3] M. Greiner, C.A. Regal, and D.S. Jin, Nature 426, 537 (2003); S. Jochim, M. Bartenstein, A. Altmeyer, G. Hendl, S. Riedl, C. Chin, J. Hecker Denschlag, and R. Grimm, Science 302, 2101 (2003); M.W. Zwierlein, C.A. Stan, C.H. Schunck, S.M.F. Raupach, S. Gupta, Z. Hadzibabic, and W. Ketterle, Phys. Rev. Lett. 91, 250401 (2003).

[4] U. Fano, Nuovo Cimento 12, 156 (1935); Phys. Rev. 124, 1866 (1961); H. Feshbach, Ann. Phys. 19, 287 (1962); S. Inouye, M.R. Andrews, J. Stenger, H.-J. Miesner, D.M. Stamper-Kurn, and W. Ketterle, Nature 392, 151 (1998).

[5] G. Baym and L.P. Kadanoff, Phys. Rev. 124, 287 (1961).

[6] G. Baym, Phys. Rev. 127, 1391 (1962).

[7] See, e.g., G. Ryckayzen, Green's Functions and Condensed Matter (Academic, New York, 1980).

[8] A. Griffin, Phys. Rev. B 53, 9341 (1996).

[9] N.M. Hugenholtz and D. Pines, Phys. Rev. 116, 489 (1959).

[10] J.R. Schrieffer, Theory of Superconductivity (Benjamin, New York, 1964).

[11] G.C. Strinati, La Rivista del Nuovo Cimento Vol.11, N.12, (1988).

[12] R. Haussmann, Z. Phys. B 91, 291 (1993). 